\title{
Microfluidic Melt Emulsification for Encapsulation and Release of Actives
}

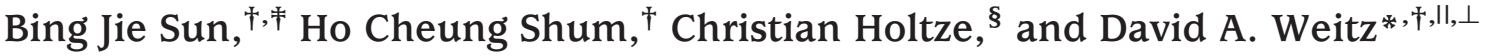

School of Engineering and Applied Sciences, Department of Physics, and Kavli Institute for Bionano Science and Technology, Harvard University, Cambridge, Massachusetts 02138, United States, The Key Laboratory of Molecular Engineering of Polymers (Ministry of Education) and Department of Macromolecular Science and Advanced Materials Laboratory, Fudan University, Shanghai 200433, PR China, and BASF SE, 67056 Ludwigshafen, Germany

\begin{abstract}
A microfluidic melt emulsification method for encapsulation and release of actives is presented. Using a water-in-oil-inwater (W-O-W) double emulsion template, solid capsules can be formed by freezing the middle shell phase. Actives encapsulated inside the solid shell can be controllably and rapidly released by applying a temperature trigger to melt the shell. The choice of the shell materials can be chosen to accommodate the storage and release temperatures specific to the applications. In addition, we have also demonstrated the same concept to encapsulate multiple actives in multicompartment capsules, which are promising as multifunctional capsules and microreactors.
\end{abstract}

KEYWORDS: emulsion $\bullet$ microfluidics $\bullet$ encapsulation $\bullet$ release

$\mathrm{D}$ ouble emulsions are structures consisting of emulsion drops of one phase containing smaller droplets of a second immiscible phase. They are widely used for encapsulation of actives ranging from food additives such as nutrients and flavors $(1-5)$, to components for personal care products $(6-10)$, to drugs for therapeutic applications (11-13). Double emulsions are thermodynamically unstable (14); for actives to remain encapsulated and thereby achieve a long shelf life, double emulsions must be stabilized and this is typically accomplished through the addition of surfactants. However, the surfactant-induced stability can also make it more difficult to destabilize the double emulsions to release the actives on demand for applications that require triggered release (15-18). This approach is especially challenging, if not impossible, for the encapsulation of actives that are themselves surface-active, as they tend to destabilize the double emulsions. In addition, another major technical challenge is the design of capsules that can co-encapsulate multiple incompatible actives or functional components. For example, echogenic liposomes for simultaneously achieving ultrasound imaging and controlled delivery have been fabricated by the co-encapsulation of a gas and a drug (19). Therefore, effective strategies to stabilize double emulsions and to achieve triggered release of actives are needed; similar strategies should be also suitable for creation of multifunctional capsules with separate compartments for

\footnotetext{
* Corresponding author. Tel: 617-496-2842. Fax: 617-495-0426. E-mail: weitz@seas.harvard.edu.

Received for review September 10, 2010 and accepted November 8, 2010

† School of Engineering and Applied Sciences, Harvard University.

* Fudan University.

$\S$ BASF SE.

"Department of Physics, Harvard University

${ }^{\perp}$ Kavli Institute for Bionano Science and Technology, Harvard University.

DOI: 10.1021/am100860b

2010 American Chemical Society
}

different components. One means of accomplishing this is to selectively gel or harden the middle fluid of the double emulsions to create solid capsules $(20,21)$. For instance, a temperature-sensitive poly(N-isopropylacrylamide) (PNIPAM) gel has been proposed for this purpose. Because PNIPAM switches between a swollen and a shrunken state at different temperatures, encapsulated actives can be released by changing the temperature (22). Another promising strategy is to solidify the middle phase by lowering the temperature so that the material undergoes a liquid-to-solid transition (23); release can then be achieved by heating the shell to melt it. This strategy of melt-emulsification, where at least one of the emulsion phases is heated above its melting point during emulsion preparation, has been demonstrated for the encapsulation of bacteria, enzymes, and FITC-BSA in lipid particles $(24,25)$ and shells of hexadecane $(14)$, respectively. However, to date, the concept of encapsulation with solidified shells has been demostrated using only conventional two-step emulsification techniques, which typically involve high shear. The lack of control over the shear leads to polydisperse emulsions; this is exacerbated in double emulsions that require two distinct steps of emulsification, each of which contributes to the polydispersity. Moreover, the high shear also promotes coalescence between droplets during the emulsification step; this leads to premature mixing of the separate drops when multiple incompatible actives are used. Thus the conventional techniques provide little control over the uniformity of the properties of the resultant capsules and prevent the co-encapsulation of multiple components in separate compartments. In particular, the release characteristics depend critically on the sizes of both the innermost drops and the shell (26). Thus a robust approach that enables fabrication of monodisperse capsules with uniform mechanical properties as well as multifunctional capsules with multiple compartments is highly desir- 


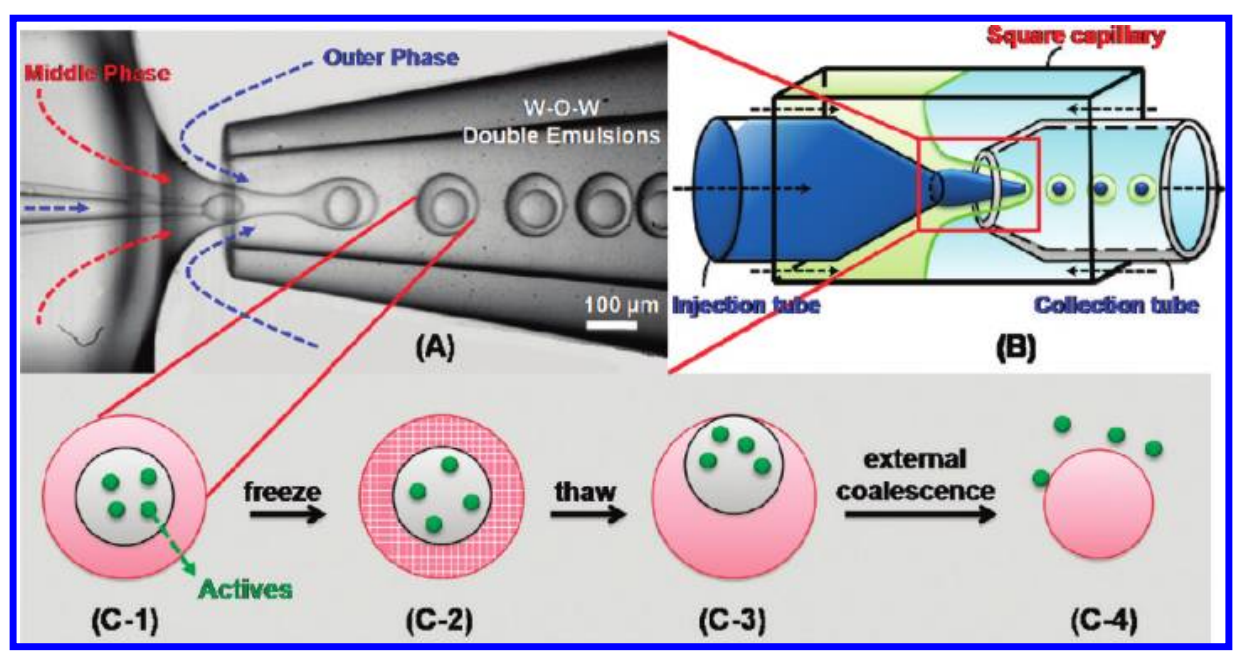

FIGURE 1. (A) Formation of monodisperse double emulsion droplets in a capillary microufluidic device with a flow focusing geometry. The continuous phase is composed of water with glycerol and PVA. The middle phase is a molten oil phase. The innermost phase contains water with glycerol and actives. (B) Schematic of the capillary microfluidic device. (C) Schematic illustrating the encapsulation and release of actives using the double emulsion-templated capsules. (C-1) shows the double emulsion droplet prepared in capillary microfluidic devices. The shell phase undergoes a liquid-to-solid phase transition upon cooling to form solid capsules shown in (C-2). By heating the capsules above the melting point of shell phase, the capsules can be thawed, forming double emulsion droplets shown in (C-3). As a result, the inner droplet within the melted shell can move freely, and because surfactants are deliberately omitted in the middle phase, actives are released upon coalescence of the inner droplet with the continuous phase, as shown in (C-4).

able; this would allow optimization of delivery characteristics for encapsulation and release of actives, as well as encapsulation of multiple incompatible actives or reactants for further chemical reactions through triggered release.

In this communication, we describe a robust microfluidic approach for fabricating solid capsules for encapsulation and triggered release of actives. Monodisperse double emulsions with a molten shell phase are prepared in a capillary microfluidic device and form solid capsules as the shell phase solidifies upon cooling. These capsules demonstrate robust encapsulation of actives independent of their size, charge, polarity, and surface-activity. Moreover, these actives can be easily released by heating the capsules above the melting temperature of the shell phase. Furthermore, to encapsulate multiple actives, we design a novel microfluidic device for forming double emulsions with multiple inner droplets, each of which contain a different active; these can be converted to multicompartment solid capsules. Such capsules are ideal for encapsulating mutually incompatible actives and reactants for further chemical reactions.

In a typical experiment, double emulsions with molten shell phases are prepared in capillary microfluidic devices, as shown in Figure 1A. The capillary microfluidic device is assembled by aligning two cylindrical capillaries coaxially inside a square capillary, as shown in Figure 1B. The fluid of innermost phase is pumped through the first cylindrical capillary or injection tube; and the middle phase flows through interstices between the outer square capillary and the capillary through which the innermost fluid is injected. The outermost phase flows into the square capillary from the opposite end. For formation of double emulsions, the molten middle phase must be immiscible with both the innermost and outermost fluids. To keep the middle phase molten, the entire device is heated to $60^{\circ} \mathrm{C}$. The outermost phase hydrodynamically flow focuses the innermost and middle phases when they meet at the entrance of the second cylindrical capillary, or collec- tion tube. Double emulsion drops are thus formed inside the collection tube, as shown in Figure $1 \mathrm{~A}$. The double emulsions are then quickly cooled below the melting temperature of the shell phase to form solid capsules. Actives encapsulated can be released on-demand by heating the capsules. The overall concept of our approach is summarized in Figure 1C.

We demonstrate this concept by using a water-in-oil-inwater $(\mathrm{W}-\mathrm{O}-\mathrm{W})$ double emulsion to prepare shells of fatty acid glycerides for encapsulating a model encapsulant, FITCDextran, a fluorescently labeled polysaccharide. We use a continuous phase of water with glycerol and poly(vinyl alcohol) (PVA), a middle phase of molten fatty glycerides (SUPPOCIRE AIM, Gattefosse, melting point (m.p.) 33-35 ${ }^{\circ} \mathrm{C}$ ), and an innermost phase of a water-glycerol mixture that contains the actives. The viscosity of the molten fatty acid glycerides is much higher than that of pure water. Thus, the range of flow rates at which double emulsions can be prepared is limited; to compensate, glycerol is added to the innermost and outermost fluids to increase their viscosities. We also add PVA to the outermost phase to stabilize the double emulsion. Double emulsion drops prepared in the microfluidic device are collected in a vial that is cooled in a bath of ice-water to speed solidification of the shells. The model active, FITC-Dextran, is completely encapsulated inside the capsules without leakage to the continuous phase, as shown by the containment of the fluorescein dyes in the inner compartments of the capsules in Figure 2A, B. These capsules remain stable at room temperature for at least six months and show no observable leakage, as demonstrated by the absence of fluorescence outside the capsules in Figure 2C, D.

In addition to the excellent capsule stability, our technique also provides easily controlled on-demand release of the actives; this is achieved by heating the capsules above the melting temperature of the fatty acid glycerides in the middle phase. To facilitate the release of actives, surfactants 


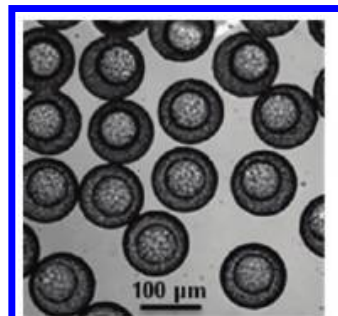

(A)

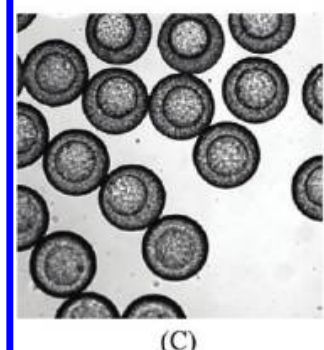

(C)

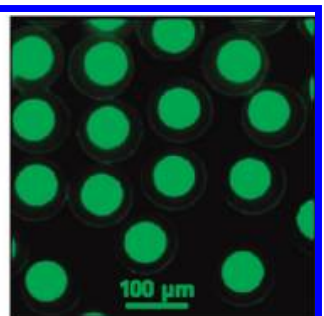

(B)

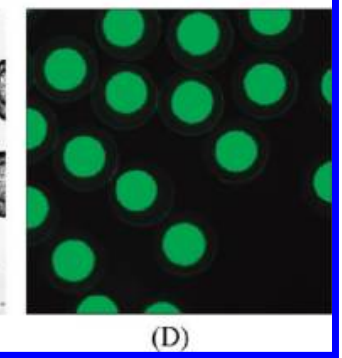

FIGURE 2. (A) Bright-field microscope image of double emulsions with solid shells of fatty acid glycerides fabricated by the microfluidic melt-emulsification method. The outer phase used in preparing the double emulsion is composed of water with $47.5 \mathrm{wt} \%$ glycerol and 5 wt \% PVA. The middle phase consists of molten fatty acid glycerides. The inner phase contains water with $50 \mathrm{wt} \%$ glycerol and $0.2 \mathrm{wt} \%$ FITC-Dextran. (B) Fluorescence microscope image of the same area as in A. (C) Bright-field microscope image of the double emulsions with solid shells of fatty acid glycerides after storage at room temperature for 6 months. (D) Fluorescence microscope image of the same area as in C.

are deliberately omitted from the middle phase so that coalescence between the inner droplets and external phase occurs very rapidly after melting of the shell. We demonstrate this mechanism by releasing $1-\mu \mathrm{m}$-diameter fluorescent latex beads encapsulated inside fatty acid glycerides capsules by heating the capsules to $37^{\circ} \mathrm{C}$. The solid shells melt upon heating; in our system, this takes about 5 mins. Upon melting, the shells undergo a solid-to-liquid transition. The inner droplets start to move freely inside the middle phase; eventually, the double emulsion drops burst, leading to the release of the latex beads, as shown in Figure 3A. Such coalescence between the inner droplets and the external phase is avoided during generation of double emulsions; this is done by rapidly freezing the double emulsion, usually within $30 \mathrm{~s}$ after fabrication. The thickness of the capsule shell layer is determined by the flow rates of the fluid phases and the diameters of the nozzles of the microfluidic devices $(27,28)$. However, as the thickness of the shell gets smaller, the inner droplets that contain the actives are more likely to coalesce with the outer continuous phase. As a result, the more stable the double emulsion is, the smaller shell thickness can be achieved. In our system, we do not apply surfactants to enable fast subsequent release of actives upon trigger; the smallest shell thickness achieved is about $10 \mu \mathrm{m}$ The encapsulation mechanism is not restricted to fatty acid glycerides; we also apply the same approach to prepare solid capsules of paraffin oil (Wako, m.p. $42-44^{\circ} \mathrm{C}$ ), nonadecane (Sigma-Aldrich m.p. $32^{\circ} \mathrm{C}$ ) and eicosane (Sigma-Aldrich, m.p. $37^{\circ} \mathrm{C}$ ). In all cases, the solid capsules exhibit similar performance as do the capsules of fatty acid glycerides. This confirms the generality of our approach and demonstrates that its applicability does not depend on the choice of the shell material.

Our technique can also be applied to encapsulate different actives. In addition to encapsulation of FITC-Dextran and $1-\mu \mathrm{m}$-diameter. fluorescent beads (Figure 4B), we apply the same technique to encapsulate model actives with different sizes and charges. For example, we use two positively charged dyes, rhodamine $B$ and toluidine blue, and a negatively charged dye, fluorescein sodium salt, as model actives; these are smaller molecules than the FITC-Dextran and the fluorescent beads. In all cases, the dyes are perfectly encapsulated in solid capsules of fatty acid glycerides, as shown in Figure 4B-D. The actives can be released by heating the solid capsules, as shown in Figure 3B. The successful encapsulation and release of the smaller dyes highlights the low permeability of the solid capsules and the effectiveness of the release mechanism.

Unlike conventional approaches, our technique is effective for the encapsulation of not just hydrophilic actives but also amphiphilic ones such as surfactants; these are typically very challenging to encapsulate using an emulsion approach. Surfactants tend to adsorb at the interfaces of emulsions and destabilize them. Using our technique, we have encapsulated a concentrated laundry detergent (Unilever), which consists of a mixture of bleach and different surfactants and is used without dilution. (Figure 4E) To demonstrate the robustness of our technique, we use a commercially available consumer laundry detergent, which is very similar to those used in a target application. Our technique differs from conventional techniques since the encapsulating shell phase solidifies before the surfactants in the detergent destabilize the emulsions, making it possible to encapsulate the surface-active amphiphilic model actives. To demonstrate the effectiveness of the encapsulation of the detergent, we mix the solid capsules with hexadecane. For perfect encapsulation, no surfactant should be present outside the capsules in the continuous phase; thus, the added hexadecane forms a layer that floats on top of the suspension of capsules, as shown in Figure $3 \mathrm{C} 1$. After heating the capsules at $37{ }^{\circ} \mathrm{C}$ for 5 mins, the detergent is quickly released from the capsules and emulsifies the hexadecane layer; this results in a cloudy mixture shown in Figure 3C2. These results highlight the effectiveness of our approach for encapsulation and release of amphiphilic actives.

To quantify the stability during storage and release efficiency of actives, we encapsulate a commercial active (BASF) in the solid capsules and monitor its release into the continuous phase using a spectrophotometer. After a month, only $5.73 \%$ of the active is released from solid capsules of fatty acid glycerides, whereas $2.93 \%$ is released from solid capsules of paraffin. The release of this small amount of actives could be due to leakage through small pores and cracks in the solid capsules as well as by diffusion through the shell layer. Despite the good encapsulation stability, actives can be released rapidly using heat as a trigger. After heating at $37{ }^{\circ} \mathrm{C}$ for 5 mins, $76.8 \%$ of the active is released from the capsules of fatty acid glycerides, whereas $55.8 \%$ 


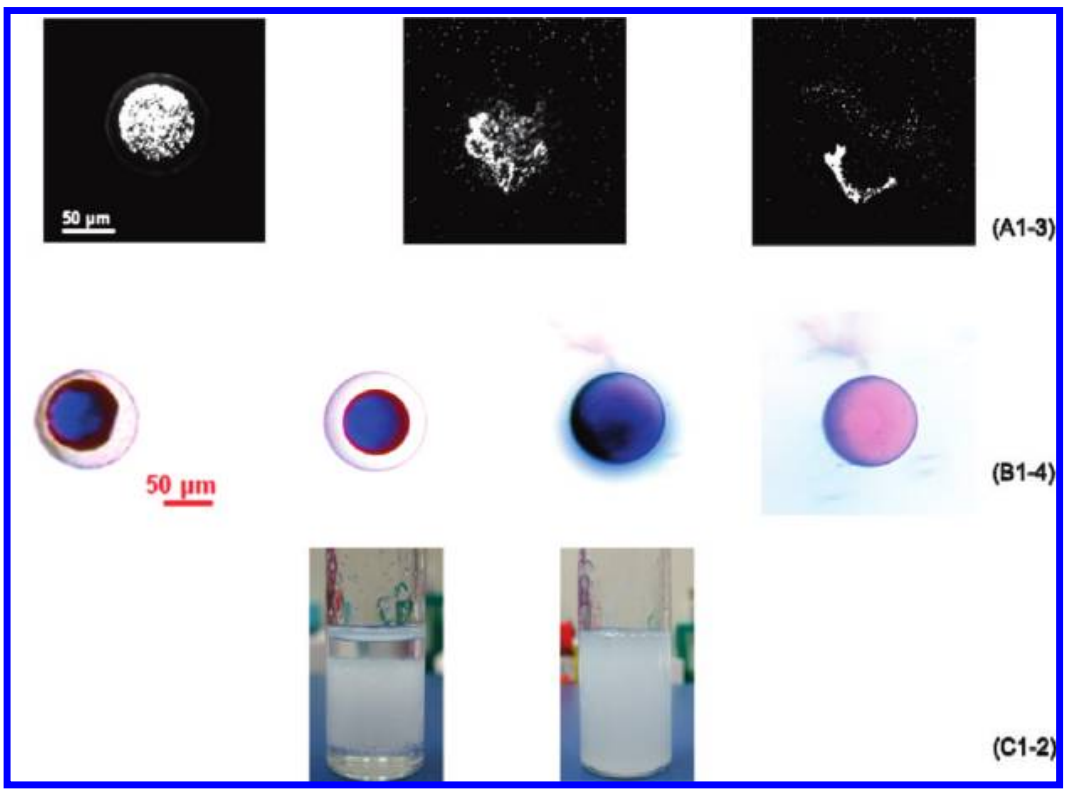

FIGURE 3. Temperature-triggered release of actives from the solid capsules. (A1-A3) Fluorescence microscope images showing the release of fluorescent beads from solid capsules of fatty acid glycerides. (A1) Solid capsules of fatty acid glycerides with fluorescent beads encapsulated at room temperature. (A2) When the capsules are heated at $37^{\circ} \mathrm{C}$, the inner droplet starts to coalesce with the continuous phase, releasing the fluorescent beads. (A3) After 5 mins of heating, the fluorescent beads are almost completely released. (B1-B4) Bright-field images showing the release of toluidine blue from solid capsules of paraffin. (B1) Solid capsules of paraffin encapsulating toluidine blue at room temperature. (B2) When heated to $45^{\circ} \mathrm{C}$, solid paraffin shell turns into a liquid shell. (B3)The inner droplet starts to coalesce with the continuous phase, releasing the toludine blue dye. (B4) Toluidine blue dyes are almost entirely released after 5 mins of heating. (C1, C2) Bright-field images showing the release of detergent from solid capsules of fatty acid glycerides for emulsification of hexadecane. (C1) The top clear layer is hexadecane, whereas the cloudy layer below consists of solid capsules with detergent encapsulated. The continuous phase of the bottom layer is a water with glycerol and PVA. (C2) After being heated at $37{ }^{\circ} \mathrm{C}$ for 5 mins, the capsules are melted, releasing the detergent to the continuous phase. The released detergent emulsifies the hexadecane above, resulting in a cloudy solution.

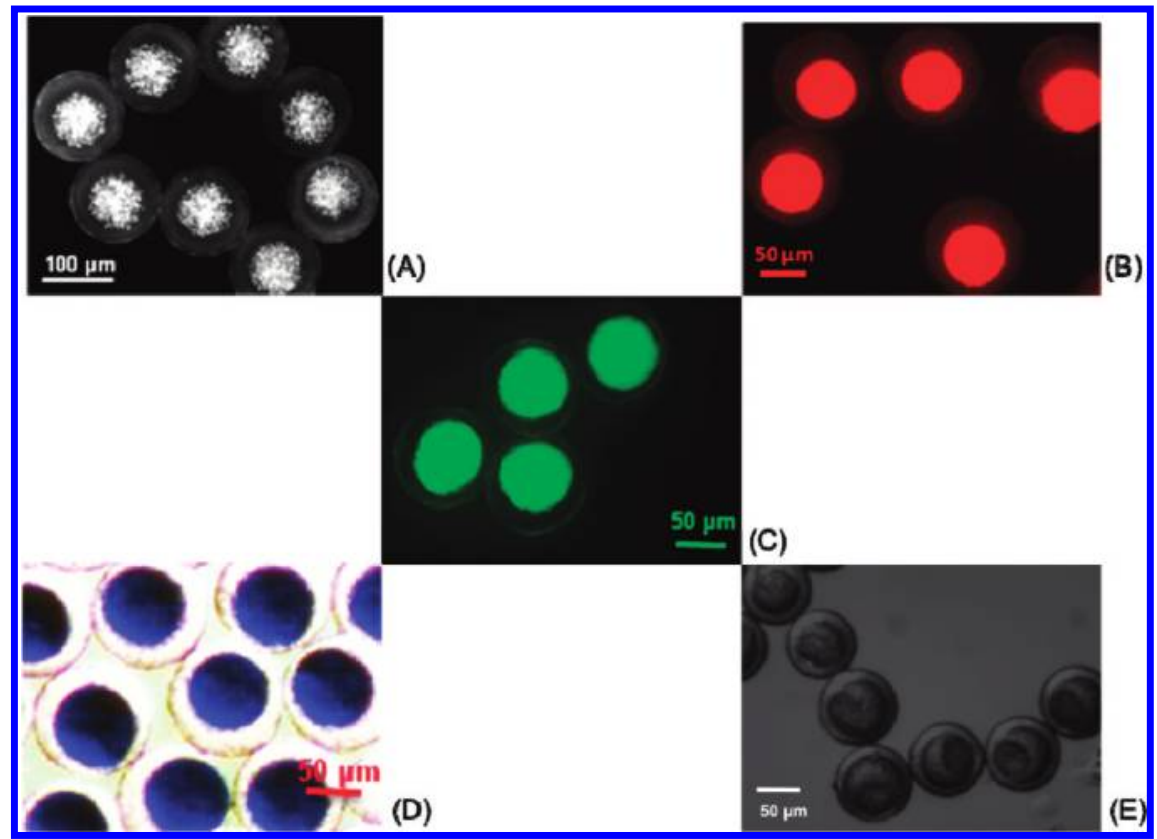

FIGURE 4. Double emulsion-templated capsules with different shells encapsulating (A) fluorescence beads, (B) rhodamine B, (C) fluorescein sodium salt, (D) toluidine blue, as well as (E) detergent.

of the active is released from the paraffin shell after heating at $45{ }^{\circ} \mathrm{C}$ for 5 mins. The results show that our approach combines excellent potential for extended shelf life with efficient on-demand release of actives. The active encapsulated here belong to a family of organic compounds commonly used as a topical drug; the efficient encapsulation and release of the active affirms the promise of the technique for controlled delivery of drugs.
An important technical challenge in this area is the coencapsulation of multiple, incompatible actives within the same structure to enable specific synergistic effects or further chemical reactions upon release. To prevent degradation or premature reaction, the actives cannot be premixed before triggered release; thus, they must be separated during the formation of the capsules. To accomplish this, we design a modified glass capillary device, which has an 


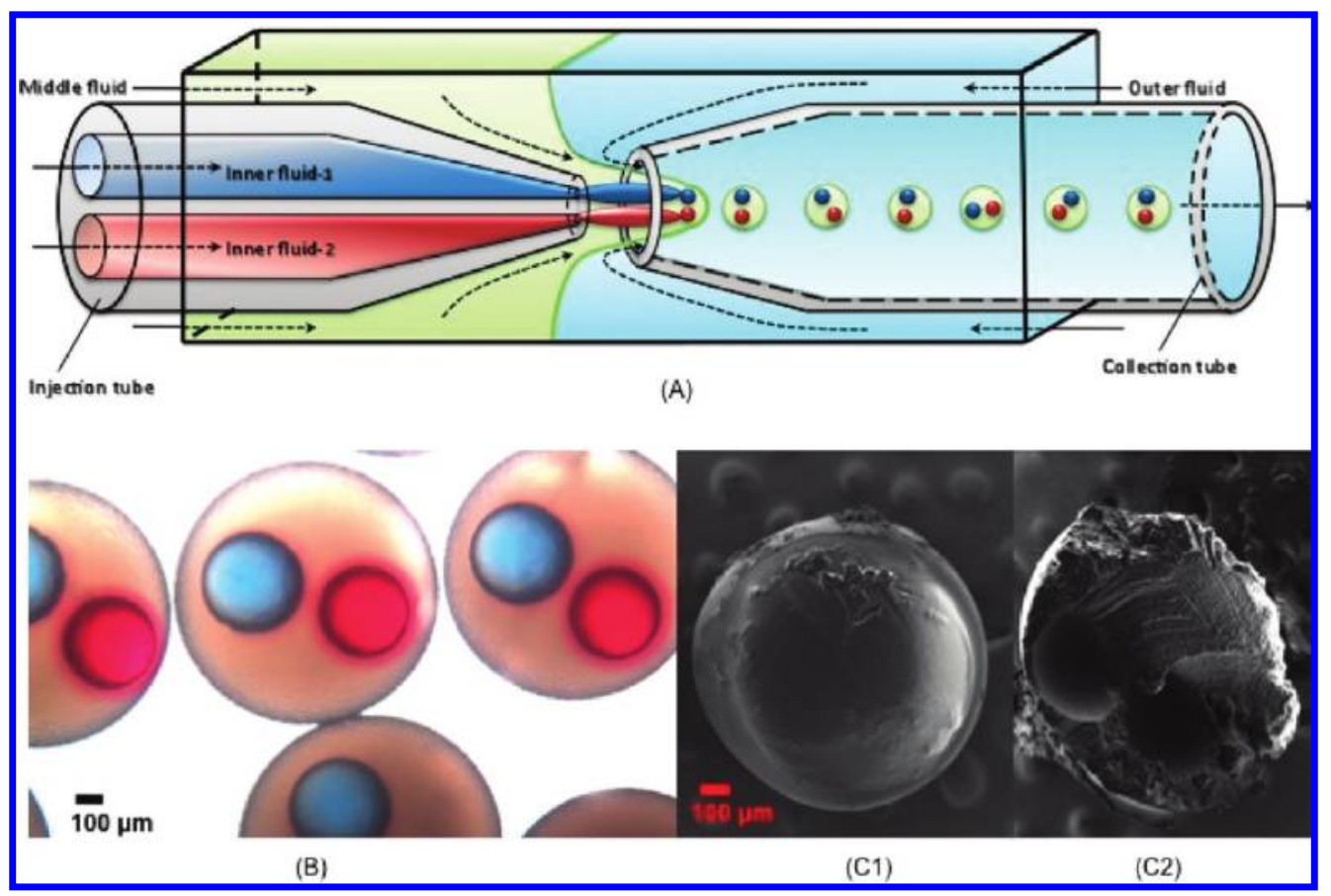

FIGURE 5. (A) Schematic of the capillary microfluidic device for generating two-bore double emulsions. The injection tube has two separate internal channels, which allow two different fluids to enter the devices separately. (B) Bright-field microscope image of the solid capsules with two inner compartments containing aqueous solutions of Wright stain (blue) and rhodamine B (red). (C) SEM images of products in B; C1 shows the surface of a dried capsule, whereas C2 shows the cross-section of the capsule.

injection tube with two separate internal channels, to fabricate solid capsules with two distinct internal compartments using the double emulsion approach. Similar double emulsions with multiple compartments have been formed in twodimensional poly(dimethyl siloxane) (PDMS) microfluidic devices (26). In this work, we use a three-dimensional glass capillary microfluidic device, which allows the use of a wide range of solvents for melt-emulsification. In the modified device, two streams of fluids with two different actives can flow separately into the device, one through each channel, as shown in Figure $5 \mathrm{~A}$. We demonstrate this using two different dyes, Wright stain (blue) and rhodamine B (red), as model actives for encapsulation in our solid capsules. The blue and red dyes flow in separate channels and form two separate inner droplets without mixing under controlled flow rates, as shown in Figure 5B, C. The inner droplets remain separate as long as the capsule is solid. By carefully tuning the separation distance between the two inner compartments, it is possible to manipulate the release profile of actives encapsulated in this fashion. If the two inner compartments are far enough apart from each other, the two actives will be released to the outer phase separately, making these solid capsules ideal for applications that require the simultaneous release of incompatible actives. However, if the two inner compartments are close to each other, they will coalesce with each other first before release to the surroundings. Thus, these solid capsules can act as microreactors in which mixing of reactants is triggered by heating. Unlike polymer capsules with multiple compartments for encapsulation (26), release of actives from these frozen capsules can be triggered upon heating. Solid capsules with multiple actives encapsulated are promising as multifunctional capsules and as microreactors.
In summary, we present a versatile technique that uses a microfluidic double emulsion approach to fabricate solid capsules for the robust encapsulation and triggered release of actives. By using a shell phase that undergoes a liquid-tosolid phase transition, double emulsion can be converted to solid capsules that exhibit excellent encapsulation efficiency and stability. Our approach for forming solid capsules does not require any complicated processing steps after generation of double emulsions drops. In addition, by omitting surfactants in the shell phase, the actives can be released very rapidly when the capsules are heated above the melting point of the shells. The robustness of this approach is highlighted by the encapsulation of amphiphilic actives, which typically destabilize emulsions and prevent encapsulation. Moreover, by combining this approach with a novel microfluidic device, we fabricate solid capsules with separate compartments for simultaneously encapsulating multiple actives; such capsules are particularly useful for encapsulating incompatible or reactive actives while keeping them separate. Our approach also provides new opportunities to design more complex capsules, for example, by using a mixture of shell materials with different properties such as melting temperatures; this will allow the manipulation of the release profiles of the actives to further control their release.

\section{EXPERIMENTAL SECTION}

Materials. Materials we used to prepare the outer phase were water (18.2 $\mathrm{M} \Omega \cdot \mathrm{cm}^{-1}$, Millipore Milli-Q system), glycerol (EMD Chemicals Inc.), poly(vinyl alcohol) (PVA; $M_{\mathrm{w}}$ : 13 000-23 000 $\mathrm{g} \mathrm{mol}^{-1}, 87-89 \%$ hydrolyzed, Sigma-Aldrich Co.). Middle phase oils included Suppocire AIM oil (mixture of glycerides of saturated fatty acids from $\mathrm{C} 8-\mathrm{C} 18$, m.p. $33-35^{\circ} \mathrm{C}$, Gatefosse'), Paraffin $\left(\mathrm{C}_{n} \mathrm{H}_{2 n+2}\right.$, m.p. $42-44^{\circ} \mathrm{C}$, Wako Pure Chemical Industries, Ltd.), nonadecane (Sigma-Aldrich Co.) and eicosane (Sigma- 
Aldrich Co.). Model actives for encapsulation were fluorescence beads ( $1 \mu \mathrm{m}$ yellow-green fluorescent sulphate microspheres, Invitrogen, Inc.), fluorescein isothiocyanate-dextran (FITCDextran, $M_{\mathrm{w}}: 10000 \mathrm{~g} \mathrm{~mol}^{-1}$, Sigma-Aldrich Co.), fluorescein sodium salt (Sigma-Aldrich Co.), toluidine blue (Fluka), rhodamine B (Sigma-Aldrich Co.), commercial active (BASF), Wright stain (Sigma-Aldrich Co.), and a consumer laundry detergent (All 2X ultra, sunshine energy, Unilever). The laundry detergent contains anionic and nonionic surfactants as cleaning agents; buffering agent, stabilizers, perfumes, brightening agents, and colorings are also added.

Microfludics. Monodisperse $\mathrm{W}-\mathrm{O}-\mathrm{W}$ double emulsions were prepared using glass capillary-based microfluidic devices $(27,28)$. The continuous phase of each encapsulation process was a mixture of water and glycerol mixed in a one-to-one weight ratio with $5 \mathrm{wt} \%$ PVA. The inner phases in various experiments included (1) water, glycerol, and FITC-Dextran $(49.9,49.9$, and 0.2 wt \%); or (2) water, glycerol, and fluorescence beads (47.5, 47.5, and $5 \mathrm{wt} \%$ ); or (3) water, glycerol, and rhodamine B $(49.97,49.97$, and $0.06 \mathrm{wt} \%)$; or (4) water, glycerol, and fluorescein sodium salt $(49.995,49.995$, and 0.01 wt \%); (5) water, glycerol, and toluidine blue (49.75, 49.75, and 0.5 wt \%); (6) water and Wright stain (99 and 1 wt \%); and (7) water and rhodamine B (99.5 and $0.5 \mathrm{wt} \%$ ). During fabrication of the double emulsion with the fatty acid glycerides, a typical set of flow rates for the outermost, middle, and innermost phases was 12000,1500 , and $200 \mu \mathrm{L} / \mathrm{h}$, respectively; with paraffin oil, the flow rates of the outermost, middle and in nermost phases were 10000,1200 , and $700 \mu \mathrm{L} / \mathrm{h}$ respectively. In the preparation of double emulsion with two inner droplets, a typical set of flow rates for the outermost, middle and the two innermost phases is 30 000, 7000, and 700 (rhodamine B)-800 (Wright stain) $\mu \mathrm{L} / \mathrm{h}$, respectively. All fluids were pumped into the capillary microfluidic device using syringe pumps (Harvard PHD 2000 series). We control the temperature of the molten phase by wrapping the syringe containing the phase with a syringe heater pad connected to a temperature controller (New Era, Inc.). This enables temperature-controlled dispensing of the fluid with $1^{\circ} \mathrm{C}$-precision. The temperature of the fluid is maintained in the device by blowing hot air over it with a light-duty heat gun (McMaster-Carr, Inc.).

Sample Characterization. The microfluidic process was monitored using an inverted optical microscope (DM-IRB, Leica) fitted with a fast camera (Phantom V9, Vision Research). Brightfield and fluorescence images were obtained with $10 \times$ objectives at room temperature using an automated inverted microscope with fluorescence (Leica, DMIRBE) equipped with a digital camera (QImaging, QICAM 12-bit). The release profile of the was monitored using a UV-vis spectrophotometer (Nanodrop, ND 1000). Scanning electron microscopic (SEM) images of dried capsules coated with a thin layer of platinum and palladium were taken using a Zeiss Supra 55VP field-emission scanning electron microscope (FESEM, Carl Zeiss, Germany) at an acceleration voltage of $20 \mathrm{kV}$

Acknowledgment. This work was supported by BASF, the NSF (DMR-1 006546), the Harvard MRSEC (DMR-0820484), and the NSFC (20676068). B.S. thanks the China Scholarship Council (20083019).

\section{REFERENCES AND NOTES}

(1) Muschiolik, G. Curr. Opin. Colloid Interface Sci. 2007, 12 (4-5), 213-220.

(2) Lobato-Calleros, C.; Rodriguez, E.; Sandoval-Castilla, O.; VernonCarter, E. J.; Alvarez-Ramirez, J. Food Res. Int. 2006, 39 (6), 678 685

(3) Weiss, J.; Scherze, I.; Muschiolik, G. Food Hydrocolloid 2005, 19 (3), 605-615

(4) Benichou, A.; Aserin, A.; Garti, N. Polvm. Adv. Technol. 2002, 13 (10-12), 1019-1031.

(5) Edris, A.; Bergnstahl, B. Nahrung 2001, 45 (2), 133-137.

(6) Lee, D. H.; Goh, Y. M.; Kim, J. S.; Kim, H. K.; Kang, H. H.; Suh, K. D.; Kim, J. W. L. Dispersion Sci. Technol. 2002, 23 (4), 491 497.

(7) Muguet, V.; Seiller, M.; Barratt, G.; Ozer, O.; Marty, J. P.; Grossiord, J. L. I. Controlled Release 2001, 70 (1-2), 37-49.

(8) Lee, M. H.; Oh, S. G.; Moon, S. K.; Bae, S. Y. L. Colloid Interface Sci. 2001, 240 (1), 83-89.

(9) Yoshida, K.; Sekine, T.; Matsuzaki, F.; Yanaki, T.; Yamaguchi, M. I. Am. Oil Chem. Soc. 1999, 76 (2), 195-200.

(10) Gallarate, M.; Carlotti, M. E.; Trotta, M.; Bovo, S. Int.I. Pharm. 1999, 188 (2), 233-241

(11) Cohen-Sela, E.; Teitlboim, S.; Chorny, M.; Koroukhov, N.; Danenberg, H. D.; Gao, J. C.; Golomb, G. L.Pharm. Sci. 2009, 98 (4), $1452-1462$.

(12) Vasiljevic, D.; Parojcic, J.; Primorac, M.; Vuleta, G. Int.I. Pharm 2006, 309 (1-2), 171-177.

(13) Nakano, M. Adv. Drua Deliverv Rev. 2000, 45 (1), 1-4.

(14) Rojas, E. C.; Staton, J. A.; John, V. T.; Papadopoulos, K. D. Lanamuir 2008, 24 (14), 7154-7160.

(15) Hanes, J.; Cleland, J. L.; Langer, R. Adv. Druq Deliverv Rev. 1997, 28 (1), 97-119

(16) Nielsen, T. F.; Ravn, P.; Bagger, Y. Z.; Warming, L.; Christiansen, C. Osteoporos. Int. 2004, 15 (2), 168-174

(17) Lazzerini, P. E.; Capecchi, P. L.; Bisogno, S.; Galeazzi, M.; Marcolongo, R.; Pasini, F. L. Ann. Rheum. Dis. 2003, 62 (7), 694-695.

(18) Creasy, G. W.; Jaffe, M. E. Ann. N.Y. Acad. Sci. 1991, 618, $548-$ 557

(19) Huang, S.-L.; McPherson, D. D.; MacDonald, R. C. Ultrasound Med. Biol. 2008, 34 (8), 1272-1280.

(20) Chu, L. Y.; Xie, R.; Zhu, J. H.; Chen, W. M.; Yamaguchi, T.; Nakao, S. L. Colloid Interface Sci. 2003, 265 (1), 187-196.

(21) Omi, S.; Katami, K. I.; Taguchi, T.; Kaneko, K.; Iso, M. L. Appl. Polvm. Sci. 1995, 57 (8), 1013-1024.

(22) Kim, J. W.; Utada, A. S.; Fernandez-Nieves, A.; Hu, Z. B.; Weitz, D. A. Angew. Chem. Int. Ed. 2007, 46 (11), 1819-1822.

(23) Guery, J.; Baudry, J.; Weitz, D. A.; Chaikin, P. M.; Bibette, J. Phvs. Rev. E: Stat. Phvs. Plasmas. Fluids, 2009, 79 (6), 060402 (R).

(24) Nori, M.; Lopes, C. M.; Favaro-Trindade, C.; Souto, E. B. New Biotechnologv 2009, 25, S56-S57.

(25) Yuan, H.; Wang, L. L.; Du, Y. Z.; You, J.; Hu, F. Q.; Zeng, S. Colloid Surf. B 2007, 60 (2), 174-179.

(26) Hennequin, Y.; Pannacci, N.; de Torres, C. P.; Tetradis-Meris, G.; Chapuliot, S.; Bouchaud, E.; Tabeling, P. Lanamuir 2009, 25 (14), $7857-7861$

(27) Shah, R. K.; Shum, H. C.; Rowat, A. C.; Lee, D.; Agresti, J. J.; Utada, A. S.; Chu, L. Y.; Kim, J. W.; Fernandez-Nieves, A.; Martinez, C. J.; Weitz, D. A. Mater. Todav 2008, 11 (4), $18-27$

(28) Utada, A. S.; Lorenceau, E.; Link, D. R.; Kaplan, P. D.; Stone, H. A.; Weitz, D. A. Science 2005, 308 (5721), 537-541.

AM100860B 